\title{
Risque et décisions en gestion de l'eau. Essai d'analyse de la rationalité du dialogue entre hydrologue et gestionnaire. [Tribune libre / Article bilingue] Risk and decision making in water resources management on the communication between the hydrologist and the decision-maker [Free opinion / Bilingual article]
}

\author{
J. Bernier
}

Volume 11, numéro 1, 1998

URI : https://id.erudit.org/iderudit/705294ar

DOI : https://doi.org/10.7202/705294ar

Aller au sommaire du numéro

\section{Éditeur(s)}

Université du Québec - INRS-Eau, Terre et Environnement (INRS-ETE)

ISSN

0992-7158 (imprimé)

1718-8598 (numérique)

Découvrir la revue

Citer ce document

Bernier, J. (1998). Risque et décisions en gestion de l'eau. Essai d'analyse de la rationalité du dialogue entre hydrologue et gestionnaire. [Tribune libre / Article bilingue]. Revue des sciences de l'eau / Journal of Water Science, 11(1), 3-24. https://doi.org/10.7202/705294ar

\section{Résumé de l'article}

Le concept de risque, trop souvent imprécis, est analysé et un essai de définition rationnelle est tenté pour tenir compte à la fois des aspects de probabilité et de conséquences dommageables. On montre que toute analyse de risque en sciences de l'eau doit résulter d'un dialogue entre hydrologue et gestionnaire qui doit respecter des critères de clarté , d'efficacité et de cohérence. On insiste particulièrement sur la nécessaire prise en compte par l'hydrologue d'une expression, même simplifiée, des conséquences dommageables réelles pour le gestionnaire ou les membres de la cible du risque. 


\title{
TRIBUNE LIBRE
}

\section{Risque et décisions en gestion de l'eau Essai d'analyse de la rationalité du dialogue entre hydrologue et gestionnaire}

FREE OPINION

\author{
Risk and decision making in water resources management \\ on the communication between the hydrologist \\ and the decision-maker
}

\section{J. BERNIER 1}

Cet article est publié intégralement en français et en anglais : les références bibliographiques sont placées zprès le texte anglais, voir $p .23^{\star}$.

This paper is published integrally in both French and English, see p. 15.

SUMMARY

The often vaguely defined concept of risk is analyzed and an attempt is made to provide a rational definition which embeds both probabilistic aspects and consequences associated with decisions. Any risk analysis in the field of water resources involves a dialogue between the hydrologist and the decision-maker, a dialogue which should be clear, efficient, and coherent. We insist in particular on the need for the hydrologist to use an expression, even simplified, of the actual damage potential, endured by the decision-maker or the people at risk.

Key-words : hydrologist, decision-maker, dialogue, probability, damage.

RÉSUMÉ

Le concept de risque, trop souvent imprécis, est analysé et un essai de définition rationnelle est tenté pour tenir compte à la fois des aspects de probabilité et de conséquences dommageables. On montre que toute analyse de risque en sciences de l'eau doit résulter d'un dialogue entre hydrologue et gestionnaire qui doit respecter des critères de clarté, d'efficacité et de cohérence. On insiste particulièrement sur la nécessaire prise en compte par l'hydrologue d'une expression, même simplifiée, des conséquences dommageables réelles pour le gestionnaire ou les membres de la cible du risque.

Mots clés : hydrologue, décideur, dialogue, risque, probabilités, dommages.

1. Professeur invité à l'INRS-Eau. Université du Québec et à l'Ecole Nationale du Génie Rural, des Eaux et Forêts. Le Pech de Biaud, 24250 Saint-Martial-de-Nabirat, France.

- Les commentaires seront reçus jusqu'au 6 novembre 1998. 


\section{1 - INTRODUCTION}

Les réflexions qui suivent concernent la notion de risque et plus spécifiquement les conditions du dialogue entre l'hydrologue et un gestionnaire de ressources en eau pour ce qui concerne les situations de risque. Les idées présentées ne sont certes pas neuves mais elles ont été rarement mises en application dans le domaine de l'eau. D'ailleurs nous réserverons des illustrations plus concrètes à un autre exposé publié ultérieurement.

Nous limitons ici les échanges à deux individus. C'est une schématisation osée du système de communication dans les études de l'eau qui est certainement beaucoup plus complexe. Nous admettrons que ce dialogue rassemble:

- un hydrologue (ou ingénieur) détenteur des informations et connaissances techniques dans les domaines de l'hydrologie, de l'hydraulique, éventuellement du calcul économique ;

- un gestionnaire (ou décideur) censé pouvoir cristalliser l'ensemble des demandes, notamment celles de protection contre "les risques " de crue ou celles de besoins en eau et pouvoir apprécier les conséquences (pas seulement économiques) des décisions qu'il doit prendre. II doit pouvoir communiquer à l'ingénieur " une expression » de ces conséquences. Nous discuterons notamment l'importance de cette partie du dialogue.

\section{2 - QU'EST-CE-QUE LE RISQUE ?}

Le Petit Larousse dit que le risque est " un danger, un inconvénient plus ou moins probable auquel on est exposé ". Tous les termes de cette définition doivent être médités et participent à la compréhension du risque. Cependant le mot "risque " est utilisé constamment dans les domaines géophysiques et de l'environnement sans que l'on sache vraiment ce que cache ce terme conçu de façon vague par les uns et les autres. On parle de risque écologique, de risque sur la santé, de risque économique ou financier, de risque d'inondation ou de séisme, etc. sans que la signification du concept soit clairement présentée et comparable d'un domaine à l'autre. En général les ingénieurs considèrent que le risque n'est défini que par sa probabilité. Hors du domaine technique, le risque (voir plus loin la perception du risque) est très souvent ressenti lorsqu'il est envisagé et distingué par ses conséquences dommageables éventuelles indépendamment de toute probabilité et dans ce cas il faut agir, dit-on, puisque le risque est censé exister! Toutes ces conceptions sont essentiellement incomplètes.

Dans la définition du Larousse « danger plus ou moins probable » signifie de fait qu'il existe un événement potentiel auquel on associe à la fois probabilité d'occurrence et conséquences dangereuses ou dommageables. Dans la définition figure aussi le concept d'exposition (d'un individu ou d'un groupe, nous dirons une cible) dans un environnement donné. Clairement cet environnement est conditionné par des choix préalables, des décisions réalisées (du gestionnaire) en matière d'aménagements hydriques par exemple. Pour caractériser une situation de risque le contexte décisionnel est nécessaire. D'ailleurs l'expression 
courante " prise de risque " souvent associée à un choix exprime bien l'ímplication dans une situation de risque résultant d'une décision.

Avant de donner une définition plus formelle d'une situation de risque, nous commencerons par illustrer le concept sur deux exemples schématiques:

- Une route sinueuse et fréquentée par des camions transportant des matières polluantes longe sur $4 \mathrm{~km}$ une rivière dont les eaux servent à alimenter une ville à l'aval. Il y a un risque que la perte de contrôle d'un camion entraîne sa chute dans la rivière polluant celle-ci. Une protection est toutefois envisageable en construisant un solide parapet sur les $4 \mathrm{~km}$ de route. Les situations possibles sont résumées dans le tableau 1.

Tableau 1 Analyse des décisions concernant l'opportunité de construction d'une protection contre la chute de camion polluant dans une rivière (les conséquences dommageables sont en $10^{6}$ trancs par exemple)

\begin{tabular}{|c|c|c|}
\hline Chute de camion dans la rivière & $\overline{\bar{E}}=$ non-chute & $E=$ chute \\
\hline Décisions & $P(\bar{E})=0,9$ & $P(E)=0,1$ \\
\hline$a_{1}$ : construction d'une protection & 5 & 5 \\
\hline$a_{2}$ : pas de protection & 2 & 50 \\
\hline
\end{tabular}

Associées à la décision $a_{1}$ (construire un parapet) et à l'événement $E$ (chute d'un camion polluant), les cases hachurées caractérisent une situation de risque ; elles font apparaître à la fois la probabilité et la conséquence de l'événement. Cette situation de risque est bien sûr très différente de celle que l'on peut associer à $a_{2}$ et à $E$ par exemple.

- On peut aussi imaginer un problème de dimensionnement d'une station d'épuration décrit par le tableau 2 où plusieurs hypothèses de capacités de traitement (les $a_{i}$ ) ainsi que de développement futur incertain de la population (les $E_{j}$ ) exprimées en équivalent-habitants sont admises. D'après ce tableau, en considerant l'événement $E$ (accroissement de population raccordée dans les 10 ans à venir supérieur à 2000 éq-hts) la situation de risque associée à $E$ de probabilité totale 0,3 et à la décision $a_{1}$ est caractérisée par les conséquences des cases hachurées.

On notera qu'en chacun de ces exemples il s'agit de conséquences monétaires dommageables de $E$ qui n'incluent pas les investissements résultant des décisions.

Tableau 2 Tableau des conséquences en $10^{6}$ francs des décisions de dimensionnement d'une station d'épuration en fonction des niveaux futur de population

\begin{tabular}{|lcccc|}
\hline \multicolumn{1}{l}{$\begin{array}{c}\text { de population sur 10 ans } \\
\text { Décisions } \\
\text { de dimensionnement }\end{array}$} & $E_{1}=500$ & $E_{2}=\mathbf{0 0 0 0}$ & $E_{3}=2000$ & $E_{4}=3000$ \\
\cline { 2 - 5 } & $P\left(E_{1}\right)=\mathbf{0 , 2}$ & $P\left(E_{2}\right)=0,5$ & $P\left(E_{3}\right)=0,2$ & $P\left(E_{4}\right)=0,1$ \\
\hline$a_{1}:$ :pas de station & 0 & 0 & 4 & 12 \\
$a_{2}$ : station de 1000 & 1 & 1 & 3 & 8 \\
$a_{3}$ : station de 2000 & 2 & 2 & 2 & 3 \\
$a_{4}$ : station de 3000 & 3 & 3 & 3 & 3 \\
\hline
\end{tabular}


Plus formellement une situation de risque est définie comme suit : un système hydrique aménagé et géré $S_{a}$ (barrages - réservoirs hydroélectriques ou autres, avec leurs évacuateurs de crue, digues, zones inondables, zones agricoles, etc.). Son aménagement et sa gestion impliquent que ce système est caractérisé par une décision préalable a (le symbole a peut représenter un "vecteur " de décisions d'aménagement et de gestion) qui a été choisie parmi un ensemble de décisions $A$. Le système est soumis à un environnement hydrologique qui peut engendrer des événements $E$ dommageables (défaillances par rapport à l'objectif fixé, inondations, ruptures de digues reliées à un événement extrême, rejets accidentels de matières polluantes, etc.).

Le système, associé à la décision a, est caractérisé par un certain nombre de variables temporelles et paramètres d'états. Parmi ceux-ci il en existe certains qui conditionnent à la fois la ou les occurrences de $E$ et leurs conséquences.

Le risque est un paramètre d'état du système et est défini par sa distribution de probabilité $P_{a}(E)$ et par une fonction de dommages résiduels quantifiés ou identifiés (après décision a) soit $D_{a}(E)$.

Dans notre premier exemple $P_{a}(E)$ ainsi que $D_{a}(E)$ sont des scalaires alors que l'événement $E$ du second exemple étant complexe, ce sont des vecteurs de probabilités et de dommages dont les éléments sont associés aux constituants de $E$. Plus généralement il peut s'agir d'occurrences répétées de $E$ où la situation de risque est définie par deux processus temporels, celui des occurrences de $E$, caractérisé par une distribution de probabilité $P_{a}(E, t)$ et celui des dommages $D_{a}(E, t)$ dépendant tous deux du temps $t$.

Pour simplifier la discussion dans la suite de ce paragraphe, on supposera que les situations de risques envisagées peuvent être comparées en considérant un simple couple de scalaires $P_{a}(E), D_{a}(E)$.

\subsection{Le risque du gestionnaire}

On remarquera que la définition précédente fait du risque un paramètre multidimensionnel caractéristique d'un système physique associé à une " décision " donnée a. Pour éviter toute confusion, on l'appellera " risque du gestionnaire " car nous considérons que c'est sa valeur (qualifiant le système réel) qui intéresse le gestionnaire une fois la décision prise. Mais c'est un paramètre incertain comme tous les autres paramètres du système liés à l'environnement hydrologique naturel, un état de la nature $\theta$ élément d'un ensemble $\theta$ au sens de la Décision Statistique (Berger, 1985). On ne peut qu'en chercher une estimation et on devra faire la même différence entre risque estimé et risque "réel " qu'en statistique classique entre estimation et paramètre.

\subsection{Le " risque " de l'hydrologue}

C'est l'hydrologue qui donne au gestionnaire les informations pour choisir la décision a, il peut même lui fournir une décision a' estimée (cas de la crue centennale par exemple) et dans ce cas son choix de a' peut être associé au " risque propre de l'hydrologue " défini à partir de ses incertitudes technologiques (liées aux erreurs de mesure des observations, à la modélisation, à l'échantillonnage, etc.) et de leurs conséquences pour l'hydrologue.

Il ne faut pas faire la confusion et le mélange des deux "risques " et pour l'éviter nous réserverons dorénavant le mot risque uniquement au risque du ges- 
tionnaire. Une fois sa décision prise, ce sont les performances réelles de son système qui intéressent d'abord le gestionnaire et non pas l'effet des insuffisances de l'hydrologue. S'il craint qu'une autre décision a" ne soit pas préférable à a', alors il devra faire comparer les deux systèmes $S_{a}$ " et $S_{a}^{\prime}$ en termes de risques du gestionnaire réels. La confusion entre aléas naturels (responsables du risque du gestionnaire) et incertitudes technologiques de la réponse de l'hydrologue doit être évitée par un traitement séparé à deux niveaux d'analyse différents.

\section{3 - L'ACCEPTABILITÉ DU RISQUE}

Considérons un ensemble de situations de risque ; chacune est donc relative à une décision a déterminée et implique des événements dommageables et caractérisés par leurs probabilités d'occurrence $P$ et leurs conséquences $D$ supposées quantifiables. $P$ et $D$ sont donc supposés ici être deux scalaires.

\subsection{Une remarque préalable}

En gestion de l'eau le risque nul (probabilité nulle) n'existe pas ; un gestionnaire ne pourra jamais que le contrôler c'est-à-dire agir de sorte à en diminuer la probabilité d'occurrence ou (et) en limiter les dommages.

Chaque situation peut être représentée par un point de coordonnées ( $P, D)$ dans le plan de la figure 1 . Bien entendu le tracé de cette figure implique que l'on soit capable de porter les dommages aussi bien que la probabilité sur des échelles numériques ou de les ordonner par rang de gravité croissante. De fait ce sera le sens qualitatif de l'analyse qui importera surtout.

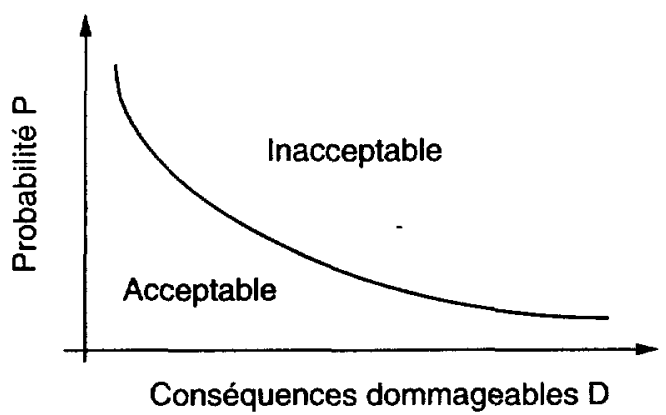

Figure 1 Représentation du risque dans le plan probabilité-dommages.

Tout gestionnaire devrait se poser la question suivante :

Un risque $(P, D)$, i.e. une situation de risque, est-il acceptable c'est-à-dire peutil laisser la décision correspondante en l'état (cette décision peut être: ne rien faire), ou est-il inacceptable c'est-à-dire doit-il modifier le risque par une action pour tendre à l'acceptabilité si possible? II est assez évident que la limite, si elle existe, entre acceptabilité et inacceptabilité doit être une courbe décroissante 
puisque toute probabilité acceptable limite est d'autant plus grande que le dommage $D$ à craindre est plus petit. Ainsi un ingénieur peut accepter la probabilité annuelle de destruction d'un pont par inondation égale à $1 / 10$ si ce pont dessert une route très secondaire mais n'acceptera pas la destruction de ce pont pour une probabilité annuelle de $1 / 100$ ou même moins s'il s'agit d'une autoroute.

Cette discussion reste assez théorique ; la représentation par la figure 1 peut cependant garder une signification qualitative si les dommages, non quantifiés, sont toutefois rangés par ordre de gravité croissante. C'est la courbe limite qui pose toutefois le plus de problèmes : elle est généralement assez floue et imprécise du fait des incertitudes multiples et aussi du fait que la perception des mêmes dommages peut varier selon les individus constituant la « cible » du risque.

\subsection{Perception du risque}

Tout individu, même possédant une culture scientifique, peut avoir et a généralement une conception de la probabilité fausse ou plutôt éloignée de celle que propose le Calcul des Probabilités classique. Par de très nombreuses expériences et enquêtes Tversky, Kahnemann, et d'autres spécialistes de ce qu'on appelle les sciences cognitives (KAHNEMANN et al., 1982), ont montré que généralement une personne appréciera la probabilité d'un événement $E$, non pas comme une fréquence ou par comparaison de paris incertains entre eux, mais en recourant à des démarches psychologiques simplifiées (dites heuristiques) appartenant le plus souvent à deux catégories :

- La représentativité (ou typicité) telle que : pour estimer la probabilité d'un événement $E$, la personne cherche si l'information disponible présente parmi tous ses aspects, au moins un aspect $E^{\prime}$ qu'elle se représente comme typique de $E$ (selon ses catégories personnelles de stéréotypes); ainsi l'occurrence de $E^{\prime}$ impliquerait presque complètement $E$. La description de crues récentes sur d'autres bassins même éloignés, peut inciter à surestimer la probabilité de crues sur un bassin particulier alors que l'information n'est pas « typique » de ce bassin donc pas transposable objectivement.

- La disponibilité c'est-à-dire la plus ou moins grande facilité avec laquelle la personne peut mobiliser des exemples semblables, par représentativité, à des cas d'occurrence de E. Ainsi la personne estimera d'autant fort la probabilité qu'un individu quelconque soit atteint d'un cancer dans une population si elle connaît des cas de maladies parmi ses proches. Il apparaît que ce dernier mécanisme joue très fortement dans l'appréciation des risques d'inondation.

L'évaluation de la probabilité d'une crue rare sur un bassin est ainsi d'autant plus surévaluée que l'occurrence (observée et subie) de la dernière crue, même beaucoup plus faible, est plus récente.

Il est important de souligner que de tels heuristiques interviennent avant toute considération de données objectives comme des fréquences observées antérieurement (même connues a priori de la personne).

Une conséquence de l'utilisation de ces heuristiques est le pouvoir d'attraction des probabilités 0 et 1 et le phénomène d'ancrage au niveau des premières évaluations quelles que soient les informations ultérieures.

Les règles les plus élémentaires du Calcul des probabilités ne sont pas vérifiées sur ces évaluations. Comme un événement $E$ et son contraire $\bar{E}$ ne sont généralement pas perçus de la même façon, l'assertion « ce barrage va diminuer 
la probabilité de submersion de $95 \%$ « est interprété différemment de la suivante : ce barrage laissera passer $5 \%$ des crues dommageables " de telle sorte que Prob(E) est différent de $1-\operatorname{Prob}(\bar{E})$.

Une autre conséquence (vérifièe expérimentalement) de la mise en œuvre de ces heuristiques est ce que Tversky appelle l'heuristique de conjonction : si $E^{\prime}$ est jugé représentatif de $E$, alors la personne jugera que Prob. $\left(E^{\prime}\right.$ et $\left.E\right)>\operatorname{Prob}(E)$. Cette inégalité est à l'opposé des règles du calcul des probabilités qui implique Prob. $\left(E^{\prime}\right.$ et $\left.E\right) \leq \operatorname{Prob}(E)$ quels que soient $E$ et $E^{\prime}$.

On dit généralement que le calcul des probabilités et la statistique donnent un cadre normatif aux problèmes de jugements face au " risque ". L'approche normative (c'est-à-dire basée sur des principes a priori) s'oppose ici à une approche descriptive qui prendrait en compte les comportements réels des individus tels que décrits ci-dessus.

On a prétendu que d'autres approches et notamment la théorie des ensembles et nombres flous et la théorie annexe des "possibilités " avaient l'avantage d'être descriptives et pouvaient être préférables à la Statistique trop normative dans ce contexte. Rien n'est moins sûr car la « rationalité de la méthode floue " demande aussi que Pos.( $E^{\prime}$ et $\left.E\right) \leq \operatorname{Pos}(E)$ ce qui est incohérent avec l'interprétation des «probabilités psychologiques » en termes de possibilités. Le flou est tout aussi normatif que la probabilité.

Les remarques précédentes, qui mériteraient certes d'être développées, avaient pour objet de montrer que la notion de risque perçu par les membres d'une cible peut être difficile à interpréter. Faut-il alors substituer ces risques perçus aux risques réels tels que définis précédemment dans une approche rationnelle au moins pour améliorer la compréhension et l'acceptabilité des décisions? Nous ne répondrons pas à cette question qui demanderait certes un débat plus approfondi.

\subsection{Retour à l'acceptabilité du risque réel}

Malgré tout des applications pratiques de l'analyse d'acceptabilité faite plus haut ont été proposées notamment dans le domaine nucléaire avec des dommages exprimés en rems, unité d'équivalent de dose pour les effets des radiations sur l'homme (FARMER, 1967). Nous l'avons surtout présentée ici car elle est à la base au moins conceptuellement des nombreuses normes ou valeurs de projets associées à des probabilités choisies forfaitairement et utilisées par les ingénieurs et décideurs.

Disons ici qu'une analyse plus complète de type économique tenant compte des coûts d'investissements de protection et de dommages peut être entreprise pour justifier le choix de la probabilité $P$ mais elle n'a été que rarement mise en œuvre concrètement compte tenu des difficultés à la fois conceptuelles et pratiques du problème. C'est cette possibilité d'éviter les difficultés en fixant des seuils de probabilité plus ou moins adaptés, qui explique la multiplicité des choix de valeurs de projet (événements décennaux, centennaux, millénaux, etc.). En fait les seuils de probabilités veulent éviter le recours à l'appréciation des dommages mais est-ce possible?

\subsection{Le modèle « inondabilité »}

II est utile de terminer ce paragraphe sur l'acceptabilité du risque par quelques commentaires sur des travaux récents du CEMAGREF concernant la ges- 
tion rationnelle des zones inondables (le modèle " Inondabilité ", OBERLIN et al., 1993). En citant les auteurs on dira que la méthode tend à " croiser connaissances des risques (en fait les probabilités ou durées de retour) et besoins de protection ». De façon plus précise elle a l'avantage de porter sur le terrain technique et probabiliste les conséquences, économiques et autres, en terme de périodes de retour acceptables. On est donc dans le cadre de la logique d'acceptabilité limite décrite ci-dessus. Mais cette méthode refuse le choix de périodes de retour forfaitairement fixées et son apport est dans la procédure complète explicitant dans chaque cas les besoins de protection des riverains en fonction d'une période de retour limite. Cette démarche se distingue des errements usuels antérieurs par l'expression des besoins de protection en terme de deux variables associées à chaque crue : le débit maximal et la durée de submersion. À ce titre elle apporte un progrès important vis-à-vis des démarches antérieures.

De notre point de vue des travaux sont toutefois nécessaires pour en améliorer la rationalité en répondant à certaines remarques. Nous en évoquerons deux ici :

- Le calcul d'une durée de retour d'un événement donné est un problème difficile s'il s'agit d'un événement associé à une entité complexe comme une crue de niveau maximal donné dès que celle-ci est caractérisée par plus d'une variable aléatoire. Par rapport à la démarche "Inondabilité " actuelle trop simplifiée à cet égard, il nous semble important de clarifier le concept de durée de retour d'une crue fonction des deux grandeurs aléatoires : débit maximal et durée.

- Les émetteurs de besoins (riverains, responsables des zones inondables) sont-ils en mesure d'apprécier la pertinence de leurs choix qui apparaissent bien techniques surtout dans la combinaison avec la période de retour admissible? Les biais psychologiques d'appréciation des probabilités s'appliquent bien entendu aux durées de retour. Éviter de tels biais psychologiques passe en partie par la clarification du concept de durée de retour aux yeux de tous les intervenants ce qui souligne l'importance de notre première remarque dont la portée nous semble dépasser le cadre de ces travaux particuliers d'ailleurs.

\section{4 - L'EXPRESSION DES CONSÉQUENCES}

Outre la signification concrète de la probabilité du risque on doit se poser une série de questions :

Peut-on éviter de croiser explicitement la probabilité et au moins une certaine évaluation des conséquences du risque?

Du point de vue hydrologique interviennent de plus les incertitudes inévitables et inhérentes à toute analyse de ces problèmes. Faut- il incorporer ces incertitudes dans le calcul et sous quelle forme les communiquer au gestionnaire?

Nous avons déjà insisté sur l'importance pour le gestionnaire de communiquer à l'ingénieur " une expression " des conséquences de ses décisions. En situations de risque et en dehors de cas simples dont nous verrons des exemples ultérieurement, le traitement complet du problème décisionnel avec évaluation précise des divers coûts est rarement possible. Est-ce à dire qu'une approche 
rationnelle, autre que les limites de probabilité forfaitaire, doit être rejetée dans ces cas où une expression quantitative des coûts des décisions est " apparemment » absente ? Nous ne le pensons pas car toute réponse au gestionnaire doit être jugée à l'aune d'un critère d'efficacité adapté et l'interprétation de ce critère en terme de coût est au moins un cadre pédagogique utile mais nous maintenons dans ces feuilles le point de vue que cette interprétation est surtout essentielle opérationnellement.

La problématique la plus fréquente est celle oủ il existe un cadre réglementaire dans lequel une autorité supérieure (au gestionnaire) impose des règlements basés sur des limites de risque (en probabilité) admissibles dans tel type de situation à risque bien déterminé. En gestion de l'eau, le problème hydrologique conséquent peut être rattaché à deux classes :

- La première concerne la prise en compte d'un risque associé à un événement $E$ de probabilité $p$. Le règlement impose de prendre en compte l'événement (c'est-à-dire construire des dispositifs de protection adéquats) si $p \geq p_{0}$. La non prise en compte signifie ne rien faire contre $E$. Le contexte décisionnel est ici réduit à 2 décisions : $a_{1}$ - prendre en compte $E, a_{2}$ - ne pas prendre en compte $E$. De tels règlements sont nombreux en industrie nucléaire notamment.

- La deuxième classe concerne l'estimation d'une valeur de projet (de protection) $\mathrm{x}_{\mathrm{T}}$ d'une variable (disons d'un débit de crue pour fixer les idées) dont la probabilité de dépassement est imposée au niveau $1 / T$ (c'est l'exemple d'une crue millénnale avec $T=1000$ ). Dans le jargon statistique $x_{T}$ s'appelle le quantile associé à la durée de retour T. Ici le contexte décisionnel est défini par l'ensemble des valeurs estimées du quantile dont chacune correspond à la décision d'assurer la protection à ce niveau.

Dans chacune des classes le règlement impose des seuils limites de probabilité ou durée de retour dont la mise en ceuvre peut paraître quelque peu abrupte mais cette logique par tout ou rien est cohérente avec l'alternative acceptable inacceptable inhérente à la démarche qui ne veut pas recourir explicitement et quantitativement aux conséquences.

Cependant nous prétendons qu'une référence au moins limitée aux conséquences du non respect éventuel des limites de probabilité est absolument nécessaire pour prendre en compte correctement l'effet des incertitudes sur le risque et sur les décisions.

Une hypothèse essentielle est bien sûr que le gestionnaire est responsable c'est-à-dire qu'il ne se contente pas d'un rôle de transmission du règlement à l'hydrologue et cherche à s'assurer que la réponse de celui-ci satisfait au mieux au règlement fixé. Au mieux signifie que gestionnaire et hydrologue se sont mis d'accord pour juger du résultat et de l'effet des incertitudes selon un critère convenablement choisi. Quel peut être ce critère?

L'hydrologue présente souvent ses estimations avec leurs propriétés statistiques traditionnelles: sans biais, variance d'échantillonnage minimale ou associées à des intervalles de confiance de seuil de confiance disons $90 \%$, etc. Ces divers critères ne peuvent prétendre satisfaire les besoins du gestionnaire. II faut veiller à bien séparer les responsabilités de chacun des intervenants. C'est confondre les responsabilités que, pour l'hydrologue, d'essayer d'imposer à un gestionnaire d'autres critères que ceux qui résultent de sa propre analyse de l'effet des incertitudes sur ses décisions concrètes. À l'opposé le gestionnaire ne peut déléguer à l'hydrologue le choix d'un critère sans lui donner au moins les élé- 
ments de ce choix. Même s'il est en présence d'un règlement supérieur à sa propre responsabilité il ne peut être neutre vis-à-vis d'une sous-estimation ou d'une surestimation (de la crue millénnale par exemple). Sans chercher à effectuer un calcul précis des conséquences, il doit traduire son attitude au moins par une " fonction de coût " instrumentale ou canonique simplifiée mais adaptée à sa situation. Dira-t-on que cette fonction de coût est arbitraire? Elle est en tout cas moins arbitraire que la seule fonction de coût quadratique à la base de la notion de variance ou des seuils d'intervalles de confiance un peu choisis comme le chiffre 13 à la roulette.

\section{5 - CONCLUSIONS}

À plusieurs reprises dans cette note l'adjectif " rationnel " est utilisé. De fait lorsqu'il s'agit du dialogue hydrologue-gestionnaire, la rationalité peut être vue de différentes façons et il importe de revenir sur la conception qui a été illustrée dans les réflexions précédentes. Pour nous la rationalité du dialogue est définie par la capacité de ce dialogue à suivre un certain nombre de principes. Bien entendu dire, dans ce cas, qu'un dialogue est rationnel n'implique pas un jugement de valeur sur ces principes. Toute rationalité n'est que relative et peut également se définir par rapport à d'autres principes. Ceux que nous inventorions ci-après ont, à notre point de vue, un intérêt opérationnel essentiel ; il s'agit de :

- La clarté du dialogue : celui-ci sera clair s'il met en évidence les responsabilités respectives de chacun des intervenants. La science et les méthodes de l'hydrologue étant souvent complexes, on met alors en avant la nécessité de les simplifier dans la communication avec le gestionnaire. On va quelquefois même jusqu'à privilégier le critère de simplicité pour le choix des méthodes d'étude elles-mêmes. Pour nous le critère de simplicité est contingent au critère de clarté et non l'inverse. Rien ne semble plus simple que le dialogue usuel où le gestionnaire demande l'estimation d'une crue de projet, disons centennale, et où l'hydrologue lui donne une valeur ponctuelle mais rien n'était moins clair dans ce dialogue particulier. La seule façon de clarifier le dialogue ici est que l'hydrologue prenne expressément en compte les conséquences pour le gestionnaire de ses propres incertitudes.

- La pertinence du choix des éléments du dialogue: ce choix concerne les objectifs à atteindre et les incertitudes technologiques éventuelles à prendre en compte. La science hydrologique étant essentiellement incertaine, par les hypothèses et modèles sur lesquels s'appuient ses résultats et par les données limitées qu'elle traite, les questions suivantes doivent être posées par l'hydrologue:

Faut-il tenir compte des incertitudes dans la réponse aux demandes du gestionnaire et si oui, sous quelle forme et comment faire un choix devant la multiplicité des incertitudes en cause?

- L'efficacité des réponses de l'hydrologue. II s'agit de répondre au mieux aux demandes du gestionnaire. Il existe bien entendu plusieurs démarches efficaces basées sur divers critères d'efficacité ; tout dépend de la façon dont on traduit les besoins du gestionnaire. Remarquons que la recherche de l'efficacité peut pousser l'hydrologue à demander au gestionnaire à reformuler sa question mais non pas à se substituer à lui pour lui imposer des critères extérieurs à ses besoins 
propres. Or certaines méthodes proposées pratiquement ne semblent pas éviter ce dernier travers que E. HALPHEN (1955) soulignait déjà avec son exemple de client acheteur de ciment (le gestionnaire) et du commerçant (l'hydrologue) ; nous citons :

"...supposons que j'ai besoin d'un ciment répondant à une certaine exigence très précise ; le commerçant à qui je m'adresse n'a pas l'article qu'il me faudrait, mais abusant de mon incompétence, il m'en glisse un autre : «Prenez cela, c'est absolument ce qu'il vous faut ". Évidemment il me vend celui de ses produits qui se rapproche le plus de mes desiderata : mais enfin ce commerçant est-il parfaitement honnête? Et sera-ce pour lui une justification suffisante de dire : " Je vous ai vendu ce ciment parce que je me refuse à tenir la qualité que vous me demandez et qui ne me plaît pas... "

En nous permettant de prolonger Halphen nous dirons que même si l'acheteur présente une demande de qualité incomplète parce qu'il croit ne pas être tenu de connaître les conséquences de son choix alors c'est au commerçant à l'interroger sur ces conséquences et lui proposer un ciment répondant à ses besoins réels, que celui-ci soit disponible en magasin ou sinon qu'il nécessite une recherche extérieure.

Un exemple de critère extérieur aux besoins d'un gestionnaire en matière de risque de crue est le critère de biais chéri par de nombreux hydrologues statisticiens. La littérature consacrée à l'étude du biais et à la recherche de méthodes les moins biaisées possibles est énorme.

- La cohérence de la démarche de l'hydrologue. Cette cohérence est assurée par le respect, à chaque étape de la démarche, des principes posés dans ses objectifs initiaux. S'agissant d'un problème où les facteurs déterminants sont aléatoires, la cohérence du respect des principes probabilistes doit être assurée. II existe (au moins) une démarche complètement cohérente : la démarche décisionnelle bayesienne. Cette démarche est maintenant bien établie dans de nombreux domaines mais mal connue dans le domaine de la gestion de l'eau au moins au niveau pratique car certains aspects des méthodologies mathématiques peuvent rebuter. On peut lui préférer des démarches pragmatiques plus simples mais attention à la cohérence d'ensemble et au danger de "changer de qualité de ciment en cours de route ». Cette préférence accordée à la simplicité est souvent motivée par la facilité de compréhension supposée par le gestionnaire. Mais la prise en compte de ce critère de préférence peut dénoter une sorte de paranoïa de l'ingénieur lorsqu'il prétend l'appliquer aux méthodes et non pas aux résultats. En expliquant les méthodes nous croyons qu'on se trompe sur la nature de l'incompréhension du gestionnaire. Nous croyons que le gestionnaire a plus besoin de comprendre le sens des réponses que lui apporte le technicien que d'assimiler la technique. La philosophie de la technique est certes importante mais la difficulté de cette philosophie n'est pas nécessairement croissante avec la difficulté (apparente) des mathématiques utilisées dans son application.

Après cet exposé un peu long, le lecteur peut se poser la question de l'opportunité et de la portée pratique d'une analyse de la rationalité des choix de décisions en matière de protection contre les crues. En fait tout décideur en ce domaine est placé dans la situation où la concrétisation pratique des effets de ses décisions n'apparaît généralement pas ou rarement même à l'échelle future de plusieurs décennies. Si rien ne se passe, peut-on dire alors que la décision était correcte ? Faut-il en conclure, comme cela s'est produit quelquefois, que la décision était trop sécuritaire et la revoir au moyen de nouvelles méthodes? A contrario s'il apparaît au moins un dommage important faut-il modifier la structure par 
de nouvelles décisions tout en sachant qu'aucune décision n'implique un risque nul ? II nous apparaît plutôt que dans ce contexte une analyse préalable de la rationalité des méthodes de calcul est la seule façon de juger de la validité des décisions et de leur impact potentiel ; elle a donc une portée pratique utile.

\section{REMERCIEMENTS}

Nous remercions chaleureusement Eric Parent de l'École Nationale du Génie Rural, des Eaux et Forêts pour ses critiques et commentaires pertinents dont la rédaction de ce texte a heureusement bénéficié. 


\section{1 - INTRODUCTION}

The following reflections concern the concept of risk and, more specifically, the dialogue between hydrologist and decision-maker in matters related to risk. The ideas presented here are not new, but have rarely been used in the field of water resources. We defer more concrete examples of applications to an upcoming paper.

The dialogue to which we refer in the following is assumed to be between two individuals. This is, of course, a simplified representation of the often complex communication systems in actual water resources studies. The dialogue involves:

- the hydrologist (or engineer) who possesses the technical information and know-how in hydrology and hydraulics, and possibly also in economical matters

- the decision-maker whose task is to make decisions that satisfy various demands, in particular those related to risk protection and water supply. The decision-maker is supposed to know the consequences (not necessarily economical) of the decisions he makes and is supposed to communicate this information to the hydrologist. We shali discuss more specifically this part of the dialogue.

\section{2 - WHAT IS RISK?}

Le Petit Larousse defines risk as "a danger, an inconvenience more or less likely to which one is exposed". Each term in this definition is important and adds to the understanding of the concept of risk. However, the term "risk" is being extensively used in the fields of geophysics and environment without a clear understanding of what it implies. We talk about ecological risk, health risk, financial risk, flood risk, earthquake risk, etc., but the meaning of "risk" is rarely defined and cannot be compared between different fields. In general, engineers define risk as a simple probability. Outside the technical fields, risk (c.f. discussion below on risk perception) is often associated with potential damage, without any reference to probability, and in such cases the general opinion is often that "actions need to be taken because a risk exists". These implicit definitions of risk are all incomplete. Larousse's definition, "danger more or less likely", signifies that a potential event exists with which one associates both an occurrence probability and a damage impact. The definition also embeds the concept of exposure of an individual or of a group, denoted the target, in a given environment. Evidently, this environment depends on some prior choices, for example decisions (made by the decision-maker) on the development of a watershed. To characterize a situation of risk, it is necessary to introduce the context of decision making. The popular expression "to take the risk" is generally associated with a choice and therefore relates consequences with a decision. 
Before giving a formal definition of a risk situation, we shall illustrate the concept by two schematic examples:

- A sinusoidal road, bordering a river for $4 \mathrm{~km}$, is extensively used by trucks transporting pollutants. The river provides water supply to a town downstream. There is a risk that an accident may cause a truck to fall into the river and pollute it. A protection is possible by the construction of a guard rail on the $4 \mathrm{~km}$ of the road. The possible situations are summarized in Table 1 . The gray fields associating the decision $a_{1}$ (construction of a guard rail) with the event $E$ (truck accident) characterize a risk situation: it involves both the probability and the consequences of the event. Clearly, this risk situation is very different from that associated with decision $a_{2}$ and E.1.

Table 1 Consequences (in millions of Francs) of constructing (or not constructing) a guard rail to protect trucks from polluting a river

\begin{tabular}{|c|c|c|c|}
\hline & Event & $\overline{\mathrm{E}}=$ "No truck accident" & $E=$ "Truck accident" \\
\hline Decision & & $P(\bar{E})=0,9$ & $P(E)=0,1$ \\
\hline$a_{1}$ : No guard rail protection & & 5 & 5 \\
\hline$a_{2}$ : Guard rail protection & & 2 & 50 \\
\hline
\end{tabular}

- Table 2 illustrates the problem of designing a water treatment plant. Several design capacities (the $a_{1}$ ) and increases in population over the next ten years (the $E_{i}$ ) are considered. According to Table 2, the event $E$ that the population increases with more than 2000 over the next ten years has a probability of 0.3 . The gray boxes correspond to the risk situation represented by the event $E$ and the decision $\mathrm{a}_{1}$.

Table 2 Consequences (in millions of Francs) of choosing a given design capacity of water treatment plant as a function of population growth.

\begin{tabular}{|c|c|c|c|c|}
\hline Population growth & $E_{1}=500$ & $E_{2}=1000$ & $E_{3}=2000$ & $E_{4}=3000$ \\
\hline Decision & $P\left(E_{1}\right)=0,2$ & $P\left(E_{2}\right)=0,5$ & $P\left(E_{3}\right)=0,2$ & $P\left(E_{4}\right)=0,1$ \\
\hline$a_{1}$ : No plant & 0 & 0 - & 4 & 12 \\
\hline$a_{2}$ : Plant with capacity 1000 & 1 & 1 & 3 & 8 \\
\hline$a_{3}:$ Plant with capacity 2000 & 2 & 2 & 2 & 3 \\
\hline$a_{4}$ : Plant with capacity 3000 & 3 & 3 & 3 & 3 \\
\hline
\end{tabular}

Note that both the above examples consider the monetary damage of the event $E$, but not the investments associated with the decisions.

We shall now give a formal definition of a risk situation. Consider the operation of a water resources system $S_{a}$ (dams, reservoirs, spillways, dikes, flood plains, agricultural areas, etc.) Its design and operation is characterized by a decision, a (a vector of design and operating decisions), chosen among the set of all possible decisions, A. The system is subject to a hydrological environment which can produce events that cause damage (failure to attain objectives, flooding, dam overflow, accidental pollution, etc.) The system, associated with the decision $a$, is cha- 
racterized by a certain number of temporal variables and state parameters. Among those are some that influence both the occurrences of damaging events and their consequences. The risk may be defined as follows: the risk is a state variable of the system which is defined by its probability distribution $P_{a}(E)$ and by a damage function which depends on the decision $a, D_{a}(E)$.

in our first example, $P_{a}(E)$ and $D_{a}(E)$ are scalars, whereas in the second example, the composite event $E$ results in vector values of $P_{a}(E)$ and $D_{a}(E)$ where each element is associated with the corresponding element of $E$. In a more general context, one can imagine several occurrences of $E$ in which case the risk is defined by two temporal processes, one representing the occurrences of $E, P_{a}(E, t)$, the other representing the associated damage, $D_{a}(E, t)$, both depending on time $t$. To simplify the following discussion, we shall assume that risk situations can be compared by considering a simple couple of scalar values, $P_{a}(E)$ and $D_{a}(E)$.

\subsection{The decision-maker's risk}

In virtue of the definition given above, the risk for a physical system is a multidimensional parameter associated with a given decision, a. To avoid confusion, we will call it "the decision-maker's risk", because this is the figure in which the decision-maker is interested upon making a decision. Note that like all other parameters in a natural environment, it is an uncertain parameter, or, in the terminology of decision theory, a state of nature $\theta$ from the set of possible states $\Theta$ (BERGER, 1985). In practice, it is estimated, and one must make the distinction between estimated risk and true risk in the same way as in traditional statistical analyses where one distinguishes estimated parameters from population parameters.

\subsection{The hydrologist's risk}

The hydrologist provides the decision-maker with information that allows him to make a decision, a. The hydrologist may in some cases provide an estimated decision, a', (for example a 100-year flood estimate) and in that case, the choice of $a^{\prime}$ is associated with "the hydrologist's risk", defined by the different technical uncertainties (measurement errors, model, sampling, etc.) and their consequences according to the hydrologist.

It is important not to confuse the two types of risk. Henceforth, whenever we use the term "risk", it is to be understood as the decision-maker's risk. Once a decision has been made, it is the actual system performance which is of primary interest to the decision-maker and not the inadequacy of the hydrologist's assessments. If the decision-maker suspects that an alternative decision $a$ is preferable to $a$ ', he should make a comparison of the two systems $S_{a}$ and $S_{a}$ in terms of the decision-maker's real risk. Confusion between natural randomness (responsible for the decisionmaker's risk) and the technological uncertainties inherent in the hydrologist's assessment should be avoided by considering two different levels of analysis.

\section{3 - RISK ACCEPTANCE}

Let us consider a set of risk situations. Each situation is described by a decision a and by damage events characterized by their occurrence probability, $\mathrm{P}$, and 
there consequences, $Q$, which are assumed to be quantifiable. $P$ and $Q$ are here assumed to be scalars.

\subsection{A preliminary note}

In water resources management, a zero-risk (zero probability) does not exist. A decision-maker can at most control the risk, that is, act in such a way as to diminish the occurrence probability of damage events and/or reduce their impact.

Each risk situation may be represented by a point $(P, D)$ in Figure 1. Evidently, a premise for the construction of such a figure is that one is able to quantify $P$ and $D$ numerically, or at least to rank them in increasing severity. In practice, the qualitative aspects of the analysis are often the main interest. Decision-makers should ask themselves the following question: is a given risk situation (P,D) acceptable? If yes, there is no need to change the associated decision (which could be "to do nothing"). If the risk is unacceptable, then it must be changed (if possible) by an appropriate action that moves the point (P,D) into the acceptable region. Clearly, the limit between the acceptable and unacceptable regions must be a decreasing curve. For example, an engineer may be willing to accept an annual probability of $1 / 10$ that a small secondary bridge be damaged by flooding, but only $1 / 100$ if the bridge serves a major highway.

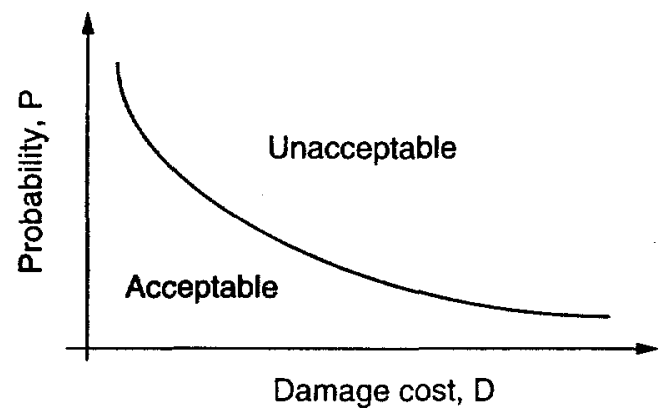

Figure 1 Representation of risk in the space of probability-damage

The above discussion has been somewhat theoretical. However, the concepts presented in Figure 1 are valid also if damages are rank simply according to severity. The curve that defines acceptable and unacceptable risk is the main problem in the analysis: it is generally very imprecise because of numerous uncertainties in the modeling, and also because of different opinions in the target group as to what is an acceptable/unacceptable level of risk.

\subsection{Risk perception}

Most individuals, including those with a scientific background, have a false perception of probability as defined and used in classical probability theory. Extensive research by Tversky, Kahnemann, and others (KAHNEMANN et al., 1982) have demonstrated that most people assess the probability of an event not by its frequency of occurrence, but by psychological (heuristic) considerations which generally fall into one of two categories: 
Representativity: To estimate the probability of an event $E$, a person seeks aspects $E^{\prime}$ in the available information which he considers representative of the event $E$. Hence, in his mind, the occurrence of $E^{\prime}$ almost entirely implies the occurrence of $E$. For example, major floods that occurred recently at some distant basins could lead to a psychological overestimation of flood risk at a given basin, although that information may not be representative for the basin and therefore not transferable in an objective sense.

Availability: The probability assessment of a person is influenced by examples, representative of the event $E$, which are available to him. For example, a person will associate a higher probability with the event that an individual gets cancer if there are cases of cancer among his close relatives. It seems that this mechanism plays an important role in the assessment of flood risk. The psychological estimation of an extreme flood event in a given basin is highly influenced by the time span to the latest occurrence of a major flood.

It is important to emphasize that heuristic assessments as those above precede any consideration of objective information such as the historically observed frequency of events (which may in fact be known by the person). A natural consequence of this is the undue interest in the probabilities 0 and $t$ and the inability of other information (data for example) to modify prior assessments. The basic rules of probability calculus generally do not apply to heuristic assessments. Since the event $E$ and its complement $\bar{E}$ are perceived differently, a statement like "this dam will decrease the flooding probability with $95 \%$ " is interpreted differently from the statement that "this dam will allow $5 \%$ of damaging floods to pass" so that $\operatorname{Pr}[E]$ is different from $1-\operatorname{Pr}[\bar{E}]$. Another consequence, proved empirically, of the application of heuristics is what Tversky denotes the heuristic of conjunction: If $E^{\prime}$ is judged representative of $E$, then a person will consider $\operatorname{Pr}\left[E^{\prime}\right.$ and $\left.E\right]>\operatorname{Pr}[E]$. This assessment is in obvious contradiction with the rules of probability calculus which state that $\operatorname{Pr}\left[E^{\prime}\right.$ and $\left.E\right] \leq \operatorname{Pr}[E]$ for any $E^{\prime}$ and $E$. Classical probability calculus provides a normative framework for risk assessment. The normative approach, which is based on a set of basic principles, therefore opposes itself to the descriptive approach which takes into account the actual behavior of individuals.

Some authors have argued that the theories of fuzzy sets and possibilities may be preferable to classical statistical theory, the latter being deemed too normative in the present context. This is not necessarily the case. The rationality of fuzzy sets theory also requires that $\operatorname{Pos}\left[E^{\prime}\right.$ and $\left.E\right] \leq \operatorname{Pos}[E]$ which is inconsistent with the interpretation of psychological probabilities measured in terms of possibilities. Fuzzy sets theory is just as normative as probability theory.

The objective of the above discussion has been to demonstrate that the risk perceived by the members of the target can be difficult to interpret. Should one substitute the perceived risk with the real risk defined previously in order to improve the comprehension and the acceptability of the decisions? This important question requires an in-depth debate which is beyond the scope of this presentation.

\subsection{Return to the acceptability of the real risk}

Practical applications of the analysis of acceptability have been done mainly in the field of nuclear power, with damage expressed in rems, a unit used to measure the effect of radiation on man (FARMER, 1967). We have presented it here, because it is the conceptual foundation of numerous regulations and design 
standards for probabilities which are used by engineers and decision-makers. Suffice it to say that a more comprehensive analysis which includes the analysis of the cost of protection and of damage could be undertaken to justify the choice of design probability $P$, but this is rarely done in practice because of the inherent conceptual and practical problems. By adopting more or less justified design standards (100-year, 1000-event, etc.), one completely circumvents the technical difficulties in determining the optimal P-value. In fact, standards aim at avoiding the assessment of potential damage, but it that reasonable?

\subsection{The INONDABILITÉ model}

We shall end this section on risk acceptability with some comments on recent research at CEMAGREF concerning the rational management of flood plains (the INONDABILITÉ model, OBERLIN et al. 1993.) According to the authors, the model aims to combine the knowledge of risk (probabilities or return periods) with the demand for protection. More specifically, INONDABILITÉ integrates into a probabilistic framework the consequences, be it economical or otherwise, and acceptable return periods. Hence, the model addresses directly the concept of maximal acceptable risk described above and avoids the use of standard design probabilities. Its main contribution is the explicit inclusion of the flood plain residents' demand for protection as a function of return period. The procedure also distinguishes itself from the classical approach by expressing the need for protection in terms of two variables associated with each flood: the maximum flow, and the duration of the submersion. However, it appears that some aspects of the model need to be further developed to improve its rationality. In particular:

- The assignment of a return period to a given event is a difficult problem if the event is complex, that is, composed of more than one random variable. The current version of INONDABILITE appears simplistic in that respect, and there is a need to clarify the concept of return period when an event is characterized by maximum flow and duration.

- Those defining the need for protection (flood plain residents, city councils, etc.) may not be able to fully understand the pertinence of their demand, which may appear very technical, for example in regard to concepts such as "admissible return period". The psychological biases which apply to probabilities of course also apply to return periods. To avoid such biases, one needs - as a minimum to clarify the concept of return period in the eyes of all those involved.

\section{4 - EXPRESSION OF CONSEQUENCES}

In addition to the very definition risk, one can ask a series of other questions, for example:

- Can the combination of probability and at least a certain assessment of consequences be completely avoided?

- Numerous uncertainties are involved in hydrological studies. Should these uncertainties be included in the analysis and how should they be communicated to the decision-maker? 
As we have already pointed out, it is important that the decision-maker communicates an "expression" of the consequences of his decisions to the engineer. In most risk situations, excluding very simple cases, an exhaustive treatment of the decision problem with an accurate estimation of the different costs is rarely possible. Should a rational approach, which goes beyond traditional design standards, be abandoned in cases where a quantitative assessment of the costs of decisions is not available? We believe the answer is no, because any answer given to the decision-maker should be judged by an appropriate measure of cost efficiency.

A common situation is when a certain standard exists in which an authority imposes admissible risk thresholds, measured in probability, for particular problems. In water resources management, two types of situations arise:

- Deciding whether or not to take into account the risk of the event $E$ with probability $P$. The standard imposes to take $E$ into account (i.e. build protection works) if $p \geq p_{0}$. The alternative is to do nothing. The decisional context is here reduced to two decisions: $a_{1}$ - take $E$ into account; and $a_{2}-$ do not take $E$ into account. Such standards are common in the nuclear industry.

- The second situation concerns the estimation of a design value, $x_{T}$, of protection, where $x$ is a variable, say the annual maximum flood, whose exceedance probability is imposed to be $1 / T$. If $T=1000$, then $x_{T}$ is the 1000 -year event. In the statistical terminology, $x_{T}$ is the quantile associated with the return period $T$. The decisional context is defined by the set of estimated quantiles corresponding to the decisions to protect at different levels.

In both cases, the standards impose threshold probabilities or return periods which may seem a somewhat rude design approach. Nonetheless, the procedure is consistent with the choice between acceptable and unacceptable risk when one is not willing to invest in a quantitative assessment of consequences. We believe, however, that some kind of assessment, even a very rudimentary one, of the consequences of violation of probability constraints is needed to correctly account for uncertainties in the risk and in the decision-making. Of course, a fundamental hypothesis is that the decision-maker is a responsible person, that is, he does not limit his decision to following the standards, but seeks to optimize his decision within the scope of the regulations. In practice, the decision-maker and the hydrologist will have to agree upon a criterion which can be used to evaluate the effects of uncertainties on decisions. Which criterion should be used? Hydrologists often present the results of an estimated value along with its statistical properties such as bias, variance, confidence intervals, etc. Such information cannot truly satisfy the needs of the decision-maker. It is important to clearly identify the responsibilities of each person in the play. For example, it is a confusion of responsibilities when the hydrologist attempts to impose on the decision-maker other criteria than those which directly relate to his own analysis of uncertainties and their impact on decisions. On the other hand, the decision-maker cannot request from the hydrologist a choice of a criterion without providing him with at least the basic implications of this choice. Even if the decision-maker is confined by regulations, he cannot be neutral as to a possible over- and under-estimation of, say, the 1000 -year flood. Although in virtue of the regulation he avoids an accurate assessment of consequences, he will need to translate his attitude by a simplified canonical cost-function adapted to the situation. Although seemingly subjective and arbitrary, such a cost-function is no more arbitrary than the quadratic loss-function which is the foundation of variance and confidence intervals. 


\section{5 - CONCLUSIONS}

Several times in the preceding discussion we have used the adjective "rational". In the dialogue between the hydrologist and the decision-maker, rationality can be viewed from different angles. In our opinion, the rationality of a dialogue should be defined in terms of the ability to base it on a set of basic principles. This definition of rationality has nothing to with whether or not the principles are reasonable. Any form of rationality is relative and can be defined in terms of other principles. The following list is, in our opinion, of fundamental practical interest:

- The clarity of the dialogue. The dialogue is clear if the responsibilities of each person involved are clearly defined. Since the scientific methods used by hydrologists are generally complex, there is a need to simplify their presentation in the communication with the decision-maker. Some times, it may even be advantageous to adopt "simple" techniques of analysis, because simplicity adds to the clarity and not vice-versa. An apparently simple example is when a decision-maker asks the hydrologist to provide a design flood estimate of, say, the 100 -year flood, and the hydrologist delivers a point estimate of this value. However, nothing is really clear in this dialogue. The only way to make the dialogue clearer is if the hydrologist takes explicitly into account the consequences (for the decision-maker) of the various hydrological uncertainties.

- The pertinence of the elements of the dialogue. The elements of the dialogue are the objectives that one seeks to attain and the uncertainties that should be taken into account in the analysis. The hydrological science abounds with uncertainties, for example related to the hypotheses and models on which the result is based and to the limited data amount available for inference. Hence, the hydrologist needs to ask himself whether the inherent hydrological uncertainties should be taken into account in the response to the decision-maker, and if yes, how they should be presented.

- The efficiency of the hydrologist's response. The ultimate objective of the hydrologist is to respond in the best possible (efficient) way to the decisionmaker's request. Of course, there may be several efficient procedures depending on the way the decision-maker's needs are interpreted. The search for efficiency may require that the hydrologist demand the decision-maker to reformulate his request, but he should not impose external criteria in addition to those relevant to the decision-maker. However, this is what often happens in practice. Halphen (1955) illustrated this by an example of a dialogue between a buyer of cement (the decision-maker) and a salesman (the hydrologist):

"Suppose that I need a cement with some specific characteristics. The salesman to whom I address myself does not have the product that I'm looking for, but, taking advantage of my ignorance, he gives me another product: "Take this! This is exactly what you need." Of course, he gives me the product in his stock that is closest to what I want. But is that salesman honest? Would it be a justification if he had said: "I have sold you this cement because I refuse to have on stock the product you were asking for."

We can extend Halphen's example and say that if the buyer asks for a product of insufficient quality because he does not know the consequences of his choice, the salesman should inquire about the use and consequences of the product and offer a suitable cement that responds to the real needs of the customer.

An example of a criterion that falls outside the scope of the decision-maker's needs in terms of risk assessment is bias, a property cherished by statistical hydrologists. The literature consecrated to the study of unbiased methods is very extensive. 
The coherence of the hydrological analysis. Coherence requires that each step in the hydrological analysis respect the principles stated in the initial objectives. When dealing with random processes where critical factors are random, the basic probability principles must be respected to assure coherence. There is at least one completely coherent procedure, the Bayesian decision theory framework. Bayesian decision analysis is today well established in many fields, but seems little used in practical water resources management, perhaps because of its mathematical complexity. More pragmatic approaches are often preferred in practice, but one should always be concerned with the coherence of the analysis and not change "the quality of cement along the road." The preference for simplicity is often motivated by the decision-maker's presumed degree of comprehension. However, we believe that what the decision-maker really needs is to understand the implications of the response given to him by the technician, rather than understanding the underlying techniques. The scientific techniques are certainly important, but it is the philosophy of the procedures that are of main interest to the decision-maker, and the complexity of the philosophy is not necessarily proportional to the mathematical complexity.

At this point, the reader may ask himself whether a study of the rationality of the choice of flood protection is warranted. As a matter of fact, most decisionmakers will never (or rarely) see the practical effect of their decisions. If nothing happens, can we say that the decision was correct? Should we conclude, as it is often done, that the decision was too conservative (too high degree of security) and revise it using new methods? On the other hand, if flooding occurs in spite of the protection, should we modify the protection knowing that no action will completely eliminate the risk? It seems that in the actual context, a preliminary analysis of the rationality of the technical methods is the only way to judge the decisions and their potential impact. Hence, the analysis of rationality suggested in this paper should be of practical interest.

\section{ACKNOWLEDGMENTS}

The author is indebted to Dr. Eric Parent, Ecole Nationale de Génie Rural, des Eaux et Forêts, whose constructive comments greatly improved this presentation.

$\mathrm{He}$ is also indebted to Dr. Peter Rasmussen, INRS-Eau, who made this excellent translation.

\section{REFERENCES}

BEARD, L.R. (1960). Probability Estimates Based on Small Normal-Distribution Samples. Joumal of Geophysical Reseach, 65(7).

BEARD, L.R. (1978). Impact of Hydrologic Uncertainties on Flood Insurance. Journal of Hydraulics Div. ASCE (HY 11), 104.
BERGER, J.O. (1985). Statistical Decision Theory and Bayesian Analysis. $2^{\text {nd }}$ Edition. Springer Verlag.

BERNIER, J., MIQUEL, J. (1975). Valeur économique de l'information nécessaire à la prise de décision en matière d'investisse- 
ment hydraulique. Proc. Congrès AIRH, Sao Paulo 1975.

BERNIER, J., Miquel, J. (1979). Incertitudes de l'information et décisions en situation de risque. Cas de problèmes de protection contre les crues. La Houille Blanche, 1.

DAVIS, D.R., KISIEL, C.C., DUCKSTEIN, L. (1972). Bayesian Decision Theory Applied to Design in Hydrology. Water Resources Research, 8(1).

FARMER, F.R. (1967). Reactor Safety and Siting. A Proposed Risk Criterion. Nuclear Safety, 8.

HALPHEN, E. (1955). La notion de vraisemblance - Essai sur les fondements du cal- cul des probabilités et de la statistique mathématiques. Publications de I'ISUP, 4(1), Paris.

KAHNEMANN, D., SLOVIC, P., TVERSKY, A. (1982). Judgment under Uncertainty: Heuristics and Biases. Cambridge University Press.

MIQUEL, J. (1984). Guide pratique d'estimation des probabilités de crues - Eyrolles

OBERLIN, G., GAUTIER, J.N., CHASTAN, B., FARISSIER, P., GIVONE, P. (1993). Une méthode globale pour la gestion opérationnelle des zones inondables: le programme Inondabilité du CEMAGREF. Sécheresse, 4. 